\title{
Encefalopatía aguda en un paciente tratado con inmunoglobulina endovenosa por un síndrome de Guillain-Barré
}

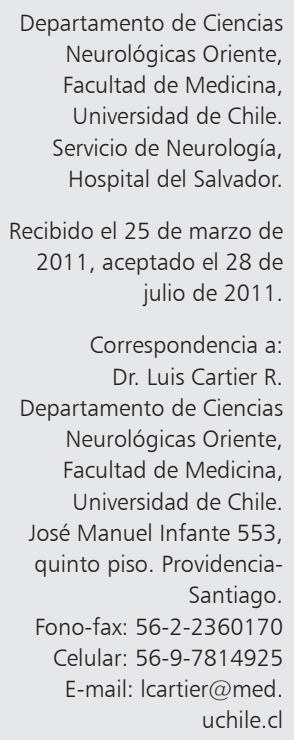

\author{
ÁLVARO SOTO V., LUIS CARTIER R.
}

\section{Acute encephalopathy associated with the use of intravenous immunoglobulin. Report of one case}

Adverse reactions to intravenous immunoglobulin (ivIg) therapy, such as anaphylaxis, acute encephalopathy, aseptic meningitis, or thrombotic phenomena are uncommon. We report a 58-year-old man with hypertension presenting with muscle weakness which led to paraparesia and respiratory failure. With the diagnosis of Guillain-Barré syndrome (GBS), he was treated with ivIg. He developed an acute encephalopathy few hours after the administration of ivIg, with a decreased level of consciousness and agitation. A CT scan revealed moderate and diffuse brain edema. Encephalopathy resolved 96 hours after ivIg withdrawal and use of plasma exchange. A CT scan performed seven days after showed the resolution of brain edema.

(Rev Med Chile 2011; 139: 1340-1343).

Key words: Guillain-Barré syndrome; Immunoglobulin intravenous; Neurotoxicity syndromes.
L a inmunoglobulina endovenosa (Igev) es un tratamiento ampliamente utilizado en una $\checkmark$ variada gama de enfermedades autoinmunes y virales ${ }^{1}$. Se usa como inmuno-modulador en dosis de 400 a $1.000 \mathrm{mg} / \mathrm{kg}^{1}$.

En neurología el uso de Igev está indicado en el síndrome de Guillain-Barré (SGB), en polineuropatías desmielinizantes crónicas (CIDP) $y$ en mononeuritis multifocales ${ }^{2}$. La eficacia del procedimiento ha sido establecida en varios estudios controlados ${ }^{3}$. Las propiedades terapéuticas se basan en el suministro de anticuerpos idiotípicos, neutralización de efectos mediados por el complemento, formación de complejos anti-membranolíticos, modulación de receptores Fc, y a efecto homeostático sobre los linfocitos $\mathrm{T}^{2}$.

El tratamiento con Igev se considera seguro. Sin embargo, entre 5 y $20 \%$ de los pacientes presentan efectos adversos menores, como mialgia, cefalea, náusea o fiebre ${ }^{4}$. Las reacciones adversas de mayor gravedad afectan al 1\% de los tratados. Se han descrito encefalopatía ${ }^{5}$, meningitis aséptica ${ }^{6}$, eventos trombo-embólicos ${ }^{7,8}$ e insuficiencia renal aguda ${ }^{9}$.

Dalacas y cols han postulado que la administración de inmunoglobulina produciría un aumento de la viscosidad de la sangre, sugiriendo que las complicaciones trombo-embólicas tendrían su origen en esta condición hematológica ${ }^{7}$. Sekul y cols, en una serie de 54 pacientes en los que usaron Igev, encontraron el desarrollo de meningitis aséptica en 6 enfermos (11\%). Ellos postularon que el mecanismo patogénico de estas meningitis sería el paso incidental de inmunoglobulinas al espacio subaracnoídeo, condición que generaría la reacción meníngea ${ }^{6}$. Por otra parte, se ha descrito una gran variedad de cambios hematológicos transitorios en relación al suministro de Igev'.

Si bien la encefalopatía aguda resulta la complicación menos frecuente, parece necesario comunicar un caso con estudio clínico completo, que podría hacer más comprensible los mecanismos patogénicos asociados a la mayoría de estas complicaciones y sugerir el manejo terapéutico en la etapa crítica. 


\section{Caso clínico}

Hombre de 58 años, tabáquico, obeso e hipertenso en tratamiento con losartán $50 \mathrm{mg}$ al día. Consultó por parestesias de manos y pies de una semana de evolución y debilidad progresiva de las cuatro extremidades que le impedían la marcha. $\mathrm{Al}$ ingreso estaba consciente y orientado, con una tetraparesia de predominio proximal, arreflexia generalizada y paresia facial derecha. Presión arterial (PA) 177/62 mmHg. Hemograma, creatinina, nitrógeno ureico, electrolitos plasmáticos, gases arteriales y pruebas hepáticas sin alteraciones. Inmunoglobulinas A, $\mathrm{G}$ y $\mathrm{M}$ en rangos normales. Con el diagnóstico de Síndrome de Guillain-Barré, se inició la administración de inmunoglobulina endovenosa $0,4 \mathrm{~g} / \mathrm{kg}$ de peso (Inmunoglobulina $\mathrm{G}$ endovenosa UNC, Argentina). Dos horas después de completada la primera infusión, desarrolló compromiso de conciencia y agitación psicomo- tora, que obligó a la sedación. Su PA era de 181/90 mmHg y se notó una agravación motora, aparición de paresia de ambos terceros pares y de pares bulbares, obligando a la intubación orotraqueal y ventilación mecánica. La tomografía computada (TC) de cerebro mostró borramiento de surcos y discreto colapso ventricular, sugerentes de edema cerebral (Figura 1). La punción lumbar dio salida a líquido cefalorraquídeo claro. Su estudio reveló 0,60 g/l de proteínas, glucosa $0,75 \mathrm{~g} / \mathrm{l}$ y 1,6 leucocitos por $\mathrm{mm}^{3}$. La electromiografía definió una polineuropatía desmielinizante aguda. Se suspendió la Igev y se inició plasmaféresis ese mismo día. Durante cuatro días permaneció comprometido de conciencia. Al quinto día fue extubado por la notable recuperación motora, que incluía la oculomotilidad, aunque persistía leve paresia de la aducción y elevación del ojo izquierdo. La TC de cerebro realizada al séptimo día, mostró regresión del edema cerebral (Figura 2) y el EEG realizado

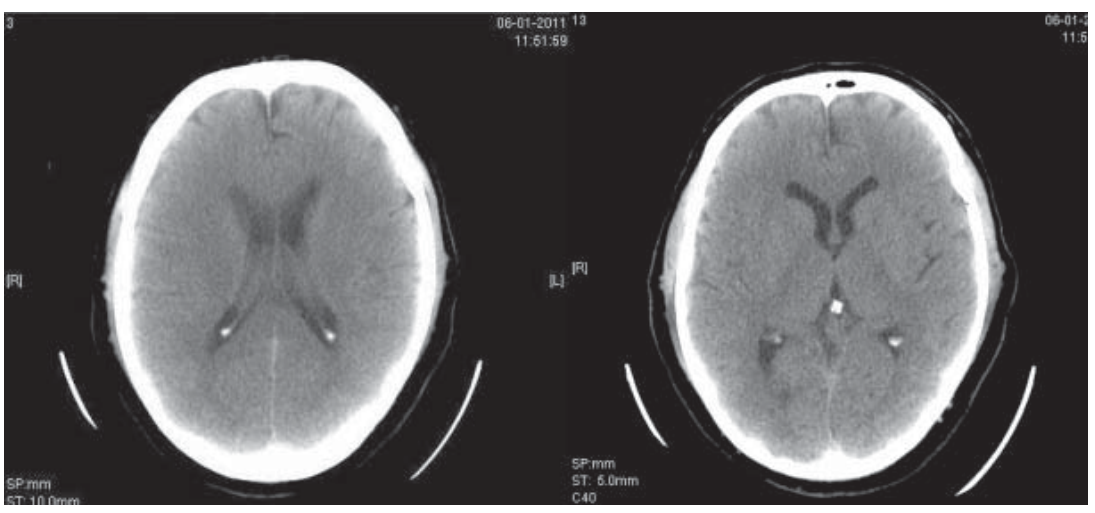

Figura 1. Tomografía computada de cerebro posterior a la administración de la primera dosis de inmunoglobulina endovenosa. Edema cerebral difuso con disminución de surcos y discreto colapso ventricular.

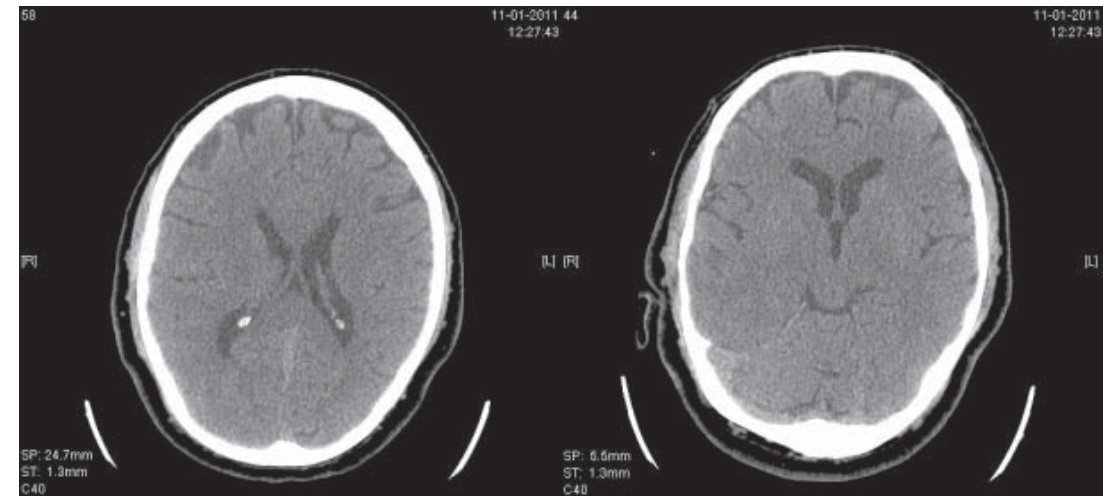

Figura 2. Tomografía computada de cerebro posterior a la suspensión de la inmunoglobulina endovenosa y la realización de plasmaféresis. Marcada disminución del edema vasogénico con mayor representación de surcos y aumento del tamaño de los ventrículos. 
ese día fue normal. Completó 5 sesiones de plasmaféresis logrando bipedestación al décimo día.

\section{Discusión}

En muchos centros, la Igev constituye el tratamiento de elección en el síndrome de GuillainBarré $^{10}$. Se han reportado reacciones precoces de intolerancia, como cefaleas, que se inician durante el primer día de la infusión y suelen controlarse con analgésicos ${ }^{4}$. Infrecuentemente, la cefalea llega a ser es muy intensa, o forma parte de una meningitis aséptica en las 72 horas que siguen a la administración de Igev ${ }^{6}$. Menos graves resultan dolores musculares u óseos, disestesias y debilidades ${ }^{4}$.

Este paciente desarrolló una encefalopatía aguda, concomitante al uso de inmunoglobulina, expresada casi inmediatamente después de la primera dosis de Igev. El compromiso de conciencia, la agitación psicomotora, la acentuación del síndrome periférico y el edema cerebral motivaron la suspensión de la Igev y el uso de plasmaféresis.

Se han reportado casos de encefalopatías vinculadas al uso de inmunoglobulinas, algunas de comienzo precoz como la observación de Harkness y cols, de una encefalopatía aguda en un $\mathrm{SGB}^{5}$, o el paciente de Mathy y cols, de una encefalopatía aguda durante la administración de Igev también en un SGB. En ambos casos se suspendió el procedimiento, regresando el cuadro ${ }^{11}$.

Además de las encefalopatías precoces, se han comunicado casos de encefalopatías tardías, que mostraron una latencia entre el término del tratamiento y la aparición del cuadro. Voltz y cols reportaron un SGB que desarrolló una encefalopatía después de completado el tratamiento con Igev, que los autores interpretaron como producto de un vasoespasmo cerebral. La resonancia magnética (RM) mostró cambios parecidos a una encefalopatía reversible posterior en la sustancia blanca de la región occipital ${ }^{12}$. Wada y cols describieron una encefalopatía en un niño de dos años. En base a estudios con RM cerebral, plantearon que el mecanismo patogénico podría atribuirse a un edema citotóxico. La técnica de difusión sugirió edema intramielínico de la sustancia blanca ${ }^{1}$.

Se han publicado SGB asociados primariamente con síndromes de encefalopatía posterior reversible (PRES), intentando relacionar patogénicamente ambos cuadros ${ }^{13-16}$. Este diagnóstico diferencial pudo haberse planteado en nuestro paciente, aunque clínicamente presentó una apariencia que lo separa nítidamente del PRES, sin crisis hipertensiva, ni disfunción autonómica, convulsiones, o alteraciones de la visión. Además, mostró edema cerebral difuso de rápida resolución, con un perfil temporal diferente. Lo mismo para la encefalopatía por Igev con latencia ${ }^{1,12}$ que pueden tener parecido radiológico, aunque en lo clínico diste mucho del PRES.

La patogenia de estas encefalopatías relacionadas con el uso de inmunoglobulinas no ha sido completamente definida. Sin embargo, en todos los casos, como en el nuestro, resulta evidente el aumento de fluidos extracelulares en el cerebro, que sugieren algún defecto en la barrera hematoencefálica, por mecanismos que parecen primariamente vasculares.

En ese sentido, Harkness ha postulado que el desarrollo de una hiperviscosidad transitoria asociada a una infusión rápida de Igev, induciría cambios en la microcirculación originando aumento de la permeabilidad capilar ${ }^{5}$. Voltz, por su parte, en su paciente con encefalopatía reversible, ha postulado el vasoespasmo como el mecanismo patogénico de la encefalopatía ${ }^{12}$. Esta hipótesis, es parcialmente concordante con la postulada para el PRES, cuya patogenia se relacionaría con la pérdida de la autorregulación circulatoria en el territorio posterior del cerebro ${ }^{13}$.

El paciente descrito presentó una reacción idiosincrática que alteró la barrera hematoencefálica, generando aumento de la permeabilidad y edema cerebral vasogénico, definido en las neuroimágenes. Estas encefalopatías y las meningitis asépticas inducidas por inmunoglobulinas tendrían una patogenia semejante. Tampoco parecería sorprendente que las frecuentes cefaleas asociadas al uso de la Igev conjuguen mecanismos vasculares ya definidos en las migrañas. Definiendo un continuo patogénico desde la simple cefalea hasta la encefalopatía aguda, perfilando un mecanismo básicamente vascular como reacción adversa primaria al uso de inmunoglobulinas ${ }^{6}$.

Tenemos la impresión que el uso de la plasmaféresis, en este caso, favoreció la rápida recuperación de la encefalopatía y del cuadro motor. Es posible que la inmediata remoción de sustancias inmuno-activas contra la pared microvascular contribuyera a ello. La buena respuesta obtenida tanto en la neuropatía como en la encefalopatía, 
sugiere que la plasmaféresis podría ser la conducta pertinente en estos cuadros.

\section{Referencias}

1. Wada A, Yoshida R, Oda K, Foyuba E, Uchida N, Kitagaki $\mathrm{H}$. Acute encephalopathy associated with intravenous immunoglobulin therapy. Am J Neuroradiol 2005; 26: 2311-5.

2. Wittstock M, Zettl UK. Adverse effects of treatment with intravenous immunoglobulins for neurological diseases. J Neurol 2006; 253 (Suppl 5): V/75-V/79.

3. Wiles CM, Brown P, Chapel H, Guerrini R, Hughes RAC, Martin TD, et al. Intravenous immunoglobulin in neurological disease: a specialist review. J Neurol Neurosurg Psychiatry 2002; 72: 440-8.

4. Bonilla FA. Intravenous immunoglobulin: Adverse reactions and management. J Allergy Clin Immunol 2008; 122: 1238-9.

5. Harkness K, Howell SJ, Davies-Jones GA. Encephalopathy associated with intravenous immunoglobulin treatment for Guillain-Barré syndrome. J Neurol Neurosurg Psychiatry 1996; 60: 586.

6. Sekul EA, Cupler EJ, Dalakas MC. Aseptic meningitis associated with high-dose intravenous immuglobulin therapy: frequency and risk factors. Ann Intern Med 1994; 121: 259-62.

7. Dalakas MC. High-dose intravenous immunoglobulin and serum viscosity: Risk of precipitating thromboembolic events. Neurology 1994; 44: 223-6.

8. Steg RE, Lefkowitz DM. Cerebral infarction following intravenous immunoglobulin therapy for myasthenia gravis. Neurology 1994; 44: 1180-1.

9. Katz U, Achiron A, Sherer Y, Shoenfeld Y. Safety of intravenous immunoglobulin (IVIG) therapy. Autoinmun Rev 2007; 6: 257-9.

10. van Doorn PA, Kuitwaard K, Walgaard C, van Koningsveld R, Ruts L, Jacobs BC. IVIG treatment and prognosis in Guillain-Barré syndrome. J Clin Immunol 2010; 30 (Suppl 1): S74-S78.

11. Mathy I, Gille M, Van Raemdonck F, Delbecq J, Depre A. Neurological complications of intravenous immunoglobulin (IVIg) therapy: an illustrative case of acute encephalopathy following IVIg therapy and a review of the literature. Acta Neurol Belg 1998; 98: 347-51.

12. Voltz R, Rosen FV, Yousry T, Beck J, Hohlfeld R. Reversible encephalopathy with cerebral vasospasm in a Guillain-Barré syndrome patient treated with intravenous immunoglobulin. Neurology 1996; 46: 250-1.

13. Van Diest D, Van Goethem JWM, Vercruyssen A, Jadoul C, Cras P. Posterior reversible encephalopathy and Guillain-Barré syndrome in a single patient: coincidence or causative relation? Clin Neurol Neurosurg 2007; 108: 58-62.

14. Bavikatte G, Gaber T, Eshiett MU-A. Posterior reversible encephalopathy syndrome as a complication of GuillainBarré syndrome. J Clin Neurosci 2010; 17: 924-5.

15. Koichihara R, Hamano S-I, Yamashita S, Tanaka M. Posterior reversible encephalopathy syndrome associated with IVIG in a patient with Guillain-Barré syndrome. Pediatr Neurol 2008; 39: 123-5.

16. Sutter R, Mengiardi B, Lyrer P, Czaplinski A. Posterior reversible encephalopathy as the initial manifestation of a Guillain-Barré syndrome. Neuromuscul Disord 2009; 19: 709-10. 